\title{
Spatial solitary-wave optical memory
}

\author{
G. S. McDonald and W. J. Firth \\ Department of Physics and Applied Physics, Strathclyde University, Glasgow, Scotland G4 ONG, UK
}

Received October 17, 1989; accepted January 26, 1990

\begin{abstract}
We consider some features of spatial solitary-wave switching in a unidirectional ring cavity that is partially filled with a fast and saturably self-focusing nonlinear medium. Large (part-beam switched) solitary arrays are considered. It is found that prescribed binary patterns may be encoded in the duration of a single cavity transit and subsequently remain stable over thousands of transits. Beam interrupt allows pixels to be switched off in fewer than ten cavity transits. Pixel instabilities on an unpixelated beam are shown to arise from spatial solitary attractive forces and intensity gradients.
\end{abstract}

\section{INTRODUCTION}

Recent research concerning all-optical switching has mainly involved the Kerr effect in nonlinear interferometers and in nonlinear couplers. ${ }^{1-3}$ Switching of solitonlike pulses in both the nonlinear interferometer and the nonlinear coupler has only recently been considered. ${ }^{4-8}$ In these cases low energy, high contrast, and stable switching have suggested solitons as the natural bits for optical information processing. Here we investigate the suitability of spatial (bright) solitary waves as pixels in a transverse binary array.

In this study we closely follow the scheme and notation of Ref. 9. The model is that of an externally pumped passive nonlinear optical ring resonator in which a wide range of spontaneously occurring spatial structures has been studied. It is of fundamental interest to examine the possibilities of control and, ultimately, utilization of these structures. Our spatial solitary waves are directly analogous to those in the temporal domain of single-mode optical fibers and arise when frequency sweeps owing to self-focusing and diffraction balance. In some specific experimental arrangements, stable self-trapped soliton beams have already been realized for the ultrafast nonlinearity of $\mathrm{CS}_{2}$, both in a three-dimensional cell ${ }^{10}$ and in a planar waveguide ${ }^{11}$ and also in glass waveguides. ${ }^{12}$ Qualitatively similar structures may also occur in atomic vapors and liquid-crystal film devices.

To achieve memory we may need to spatially modulate the medium or the input light or both. Medium pixelation leads to rather involved considerations, as can be illustrated by particular examples cited from the physics of nonlinear interference filters, where both analytic ${ }^{13}$ and experimental ${ }^{14}$ research has been performed. In light of this, we shall examine pixelation through spatial modulation of only the pump field. Our work, therefore, broadly continues on from publications that dealt with bistable arrays induced in a nonlinear Fabry-Perot resonator. ${ }^{15}$ Those studies modeled the specific physics of InSb étalons in which diffusive coupling has been seen to be the dominant transverse effect. ${ }^{16}$ Here we study the case of dominantly diffractive coupling and seek to establish device prospects and limitations for this kind of interpixel cross talk.
Neglecting diffraction permits much analytic progress to be made, whereas its inclusion results in a relatively intractable system for which full numerical simulations are necessary. It is hoped that, in the self-focusing case, the attracting nonlinear basis (solitary waves) will simplify the spatial complexity to consideration of only these (natural) nonlinear modes. A study of two-beam coupling has been performed on a similar system by considering a single, low Fresnel number in the self-defocusing regime. ${ }^{17}$ Here we present results from a two-beam coupling investigation with a self-focusing nonlinearity and in a model that neglects saturable absorption. This generalizes the earlier results ${ }^{17}$ and provides a necessary underpinning for our array studies.

\section{THEORY}

The complex field $G_{n}(x, z)$, during its $n$th transit through the medium, may be described by a nonlinear Schrödingertype evolution equation:

$$
2 i \frac{\partial G_{n}}{\partial z}+\frac{\gamma}{p} \frac{\partial^{2} G_{n}}{\partial x^{2}}+f\left(\left|G_{n}\right|^{2}\right) G_{n}=0 .
$$

We consider a medium whose response is much faster than the cavity transit time, which thus permits adiabatic elimination of the polarization from the Bloch equations. Planar waveguide geometry reduces the diffractive Laplacian to an operator in one Cartesian dimension. Cavity boundary conditions complete a full transverse, and indeed infinite-dimension, generalization of the map that was considered by Ikeda ${ }^{18}$ :

$$
G_{n+1}(x, 0)=A(x)+R \exp \left(i \Phi_{0}\right) G_{n}(x, p) .
$$

The pump field, denoted $A(x)$, is sufficiently detuned from two-level atom resonance to furnish a purely dispersive nonlinearity, $f\left(\left|G_{n}\right|^{2}\right)=-1 /\left(1+2\left|G_{n}\right|^{2}\right)$, though here we do not consider the extreme Kerr limit. The scaling of the propagation and transverse dimensions (see Fig. 1) is such that we deal with an effective medium length, $p=\alpha_{0} L_{1} / \Delta$, whereas our (beam) Fresnel number, $F=$ $n_{0}\left(\omega_{1 / 2}\right)^{2} /(\lambda L)$, is scaled into $\gamma=\ln 2 /(4 \pi F)$. Parameters $\Phi_{0}, \Delta$, and $\alpha_{0}$ are the cavity mistuning, pump detuning, and medium linear absorption coefficient, respectively. 


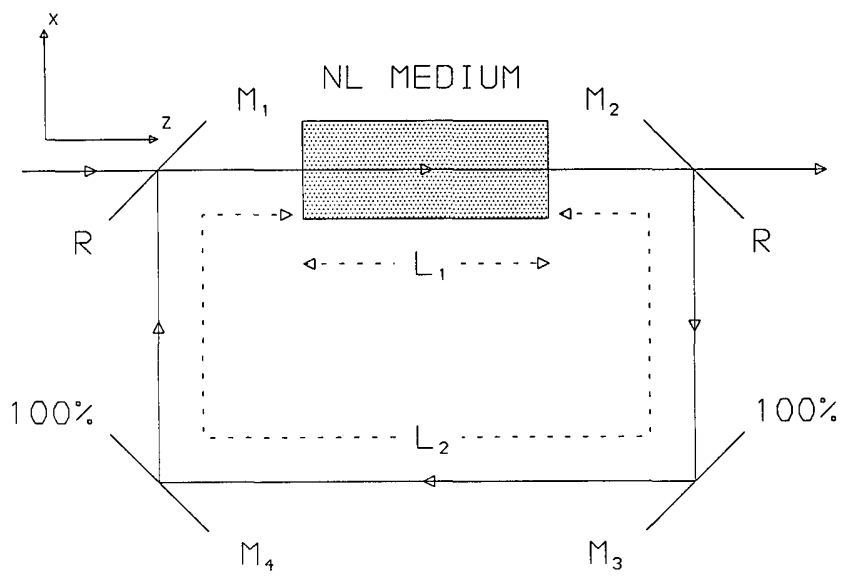

Fig. 1. Schematic representation of our optical resonator, which is partially filled with a nonlinear medium. The laser beam(s) enters through $\mathrm{M}_{1}$, which, like $\mathrm{M}_{2}$, has an intensity reflectivity of $R=0.9$. Output through $\mathrm{M}_{2}$ is monitored.

We normally allow for the medium to fill only part of the cavity; we perform linear lossless propagation in the remainder. In the simulations presented, an integration is first made to the output mirror $\mathrm{M}_{2}$ to permit data collection before completion of the resonator circuit. The beam path drawn in Fig. 1 corresponds to the $x=0$ axis. Since we use plane mirrors, beams may circulate in the cavity on both sides of this axis. In all cases $p=2 \eta$ and $\Phi_{0}=0.4 \eta$, where $\eta=+1$ (a self-focusing medium). Our chosen value of $\Phi_{0}$ permits a wide bistable loop and avoids Ikeda-type instabilities. Integrations within the medium are performed by using the efficient split-step Fourier operator method. ${ }^{19}$

\section{TWO-BEAM COUPLING NUMERICAL EXPERIMENTS}

To investigate parallel operation of two all-optical switches, we choose to use a low Fresnel number $(F=1)$ and hence stay just within the whole-beam switching regime. ${ }^{20}$ Here the (natural) solitary-wave width is of the order of a pump beam width. The form of $A(x, t)$, in this case, is

$$
\begin{aligned}
A(x, t)= & a_{01} \exp \left[-\left(x-x_{1}\right)^{2}\right] \\
& +a_{02}(t) \exp \left[-\left(x+x_{1}\right)^{2}+i \Delta \Phi\right] .
\end{aligned}
$$

Hold-beam separation will be measured in units of intensity, full width at half-maximum, so we define $X 0=$ $(2 / \ln 2)^{1 / 2} x_{1}$. Temporally, both beams are at a working point $a_{H}$, while $a_{02}(t)$ encompasses an additional square pulse of amplitude $a_{S}-a_{H}$ on the left-hand beam. These amplitudes are parameterized around the peak input amplitudes for switching up and down $\left(a_{\wedge}\right.$ and $a_{\vee}$, respectively) and are given as

$$
a_{H}=a_{\vee}+c_{H}\left(a_{\wedge}-a_{\vee}\right), \quad a_{S}=c_{S} a_{H} .
$$

In this system, parameter values $c_{H}=0.75$ and $c_{S}=1.5$ supply safe hold points, free from overshoot and undershoot switching, and necessitate only modest amplitude and duration of address. An overall phase difference $\Delta \Phi$ between the pump beams is also included. Such a factor may arise, for example, owing to beam multiplexing. The key problem is determining under which values of separation and relative phase of the pumps the left-hand beam can be independently switched.

Figure 2 gives examples of simulations in the selffocusing case. The dashed curves are the prescribed hold beams, whereas the resonator output profiles are drawn solid. For $X 0=2.0$ and $\Delta \Phi=0.0$, we found that switching of the left-hand beam also triggers the right-hand beam, after which the beams are in phase with each other and coalesce in the final plot. However, for $X 0=2.25$, the left-hand beam can be selectively addressed. In Fig. 2(a) output before, during, and after the address pulse is shown for the latter case. During address the lefthand beam lies outside the bistable region. After removal of the switch it relaxes to a point on the upper branch. A slight drop in amplitude of the right-hand beam occurs because of the finite amount of cross talk from the switched-on beam, which experienced a phase change on transition to the upper branch of approximately $\pi$ rad. For $X 0=2.00$ and $\Delta \Phi=\pi$, Fig. 2(b), the relative beam phase again causes a degree of mutual annihilation, this
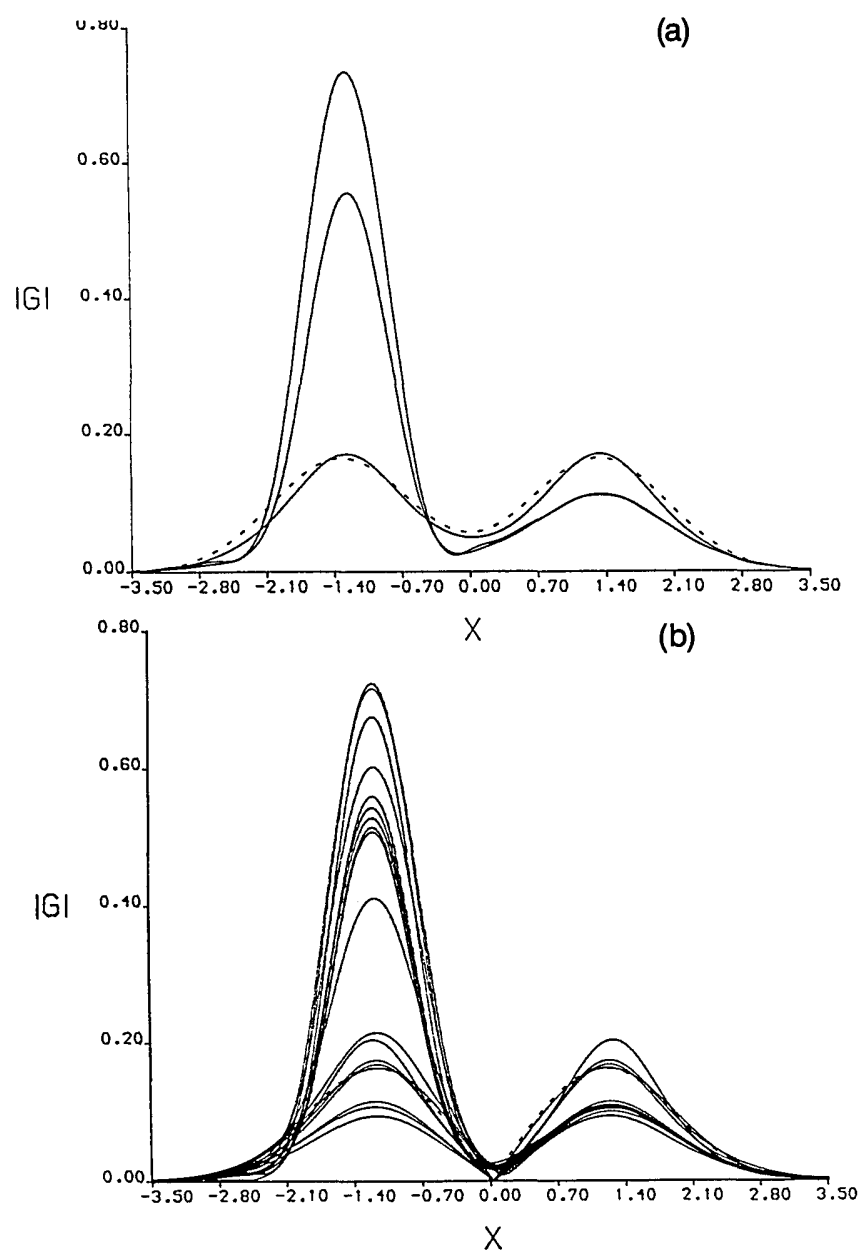

Fig. 2. $\eta=+1$. (a) Spatial profiles before, during, and after the left-hand site has been addressed. Also shown (dashed curve) is the input, $A(x)$. This full width at half-maximum (intensity) separation of hold beams, $X 0=2.25$, is required for independent switching to be maintained when $\Delta \Phi=0.0$. (b) Pixel independence at $X 0=2.0$ is achieved through an overall phase differential $(\Delta \Phi=\pi)$. Here some intermediate profiles are also given. 
time reducing peak beam amplitude before switching. This restraint, in conjunction with the intermediate destructive phase barrier, halts the switching wave and permits independent switching down to an impressive $X 0=1.25$.

In Fig. 2(b) intermediate profiles (every fifth round trip) are given that show typical temporal dynamics for these simulations. One can see that instantaneous, and hence abrupt, initialization of the hold beams results in a significant overshoot from the (lower-branch) equilibrium point and that transient switching up and relaxation oscillations occur on a time scale of two or more plot periods. The time taken for these processes may be attributed to the whole-beam nature of the solutions and should be kept in mind when part-beam arrays are considered below. In particular, the conventional wisdom that fast switching occurs only in the whole-beam regime ${ }^{20}$ should be challenged.

The corresponding investigations for the self-defocusing nonlinearity have produced less encouraging results and an additional instability. ${ }^{21}$

\section{OPTICAL SOLITARY-WAVE MEMORY ARRAYS}

As we have seen, the interaction of adjacent input Gaussian beams is rather complex and sensitive to the relative phase of these pump fields. For extension to a large number of pixels, an attractive strategy is to attempt to use the spontaneous solitary-wave structures observed in switching a single, and thus implicitly coherent, broad input beam. 9,21 Each peak, which we will henceforth term a soliton, suggests itself as a natural pixel, and our goal is to establish criteria under which information can be encoded in the ring cavity system in terms of the number and location of spatial solitons. The first point to note is that the soliton structures observed in spontaneous switching are analogous to bit strings of the form ...11111..., while the lower branch (no-soliton) state corresponds to $\ldots 00000 \ldots$ So far, then, we still have only a two-state memory; to store information we have to examine ways of creating and maintaining subsets of the $m$ solitons that fill the switched-on portion of the beam.

Two strategies suggest themselves: One is to attempt to create different numbers of solitons by spatially selective address pulses and to examine their stability against coalescence and collapse. If we could create any number $0,1, \ldots, m$ of such solitons in a given system, we can clearly store any of the first $m$ integers or equivalent information. Figure 3 shows what happens when two solitons are created on a broad Gaussian pedestal. They drift toward each other and eventually coalesce, emitting (nonsoliton) radiation transversely. That this is not inevitable is shown in Fig. 4, where two solitons on a plane-wave background appear to be stable. A detailed account of these studies will be given elsewhere.

The second, more promising, strategy is to assign significance to the positions, not just to the number, of solitons present, just as in the binary representation of numbers. An $m$-soliton beam can then represent $2^{m}$ binary numbers. We must be sure, however, that the solitons do not drift around, since this would corrupt the information stored. If we view the soliton as a pair of switching waves, it is clear that a spatial modulation of the hold beam may stabilize their position. To this end, our

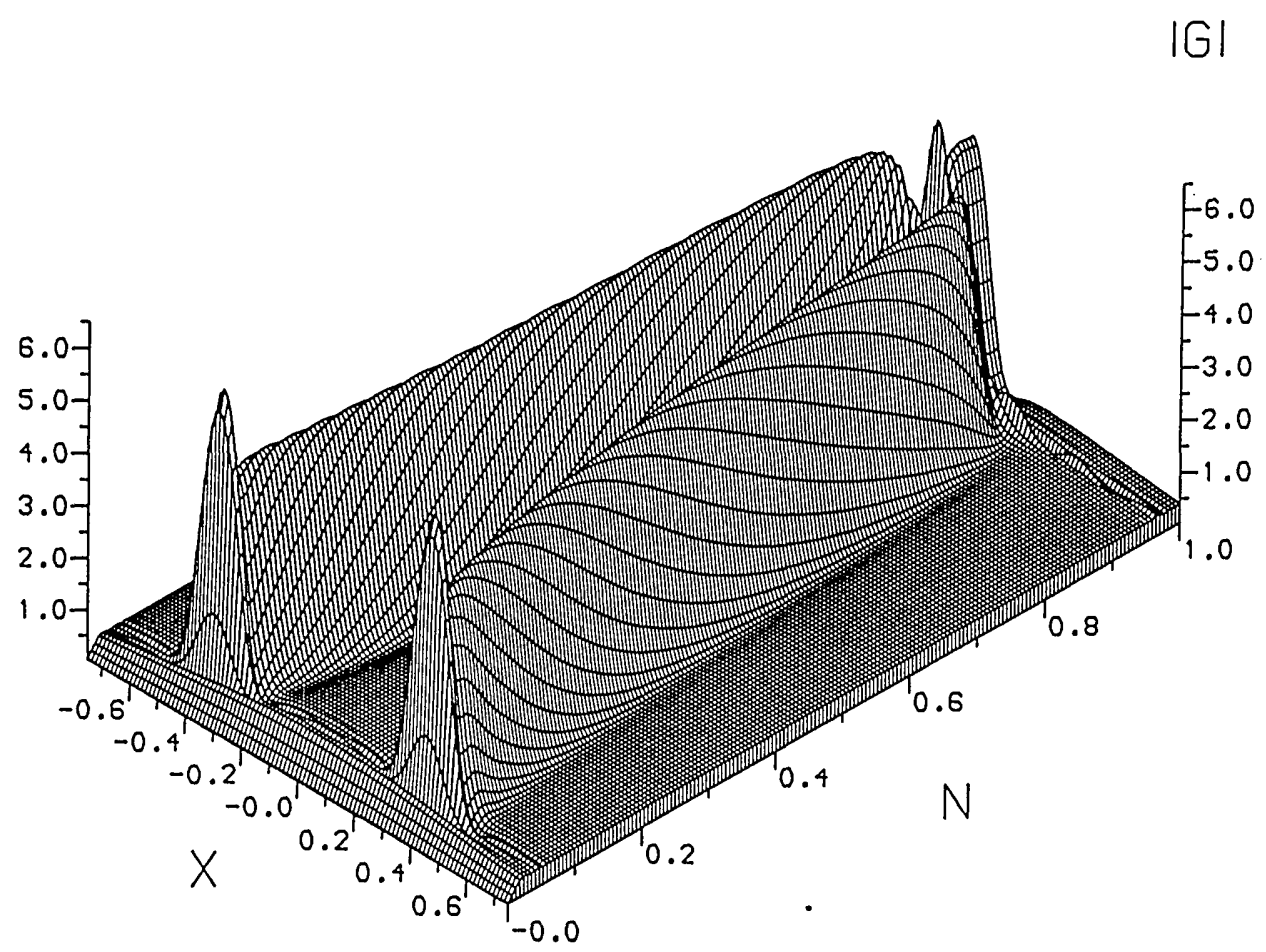

Fig. 3. Spatial motion due to (unbalanced) nonlinear index differentials. Two solitons are symmetrically induced on an $F=800$ beam. Subsequent evolution displays a migration of these waves toward the beam center and ultimate coalescence. $N$ is in units of 1000 cavity transits, $|G|$ axis $\times 10^{-1}$. 


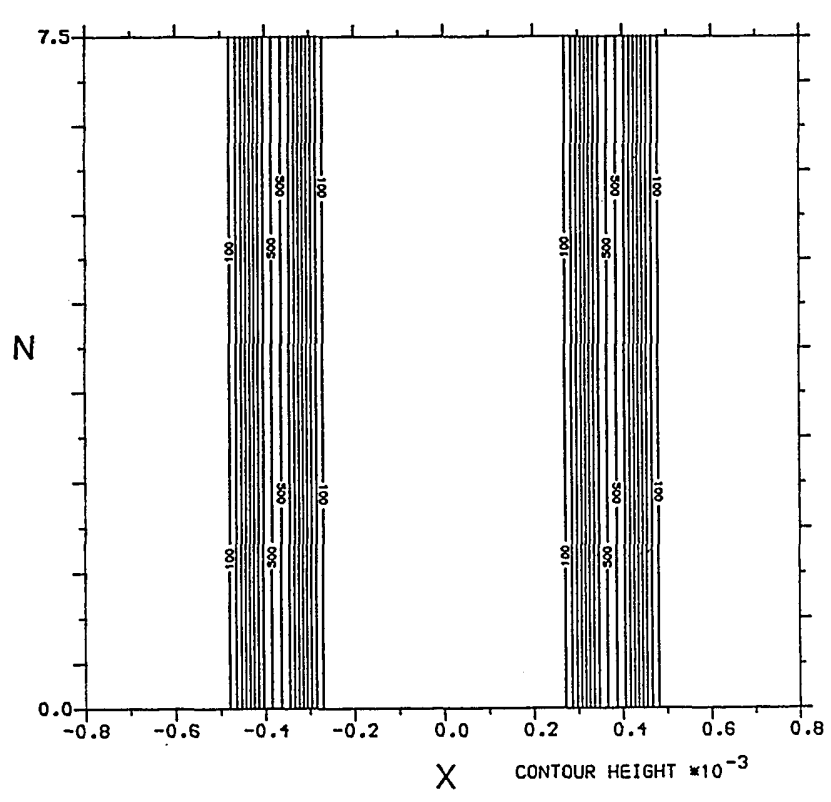

Fig. 4. For a separation identical to that used in Fig. 3, a (spatially) periodic plane-wave simulation is performed. Solitonsoliton coupling is increased here $(F=50)$, and no significant migration is observed. $N$ is in multiples of 1000 transits.

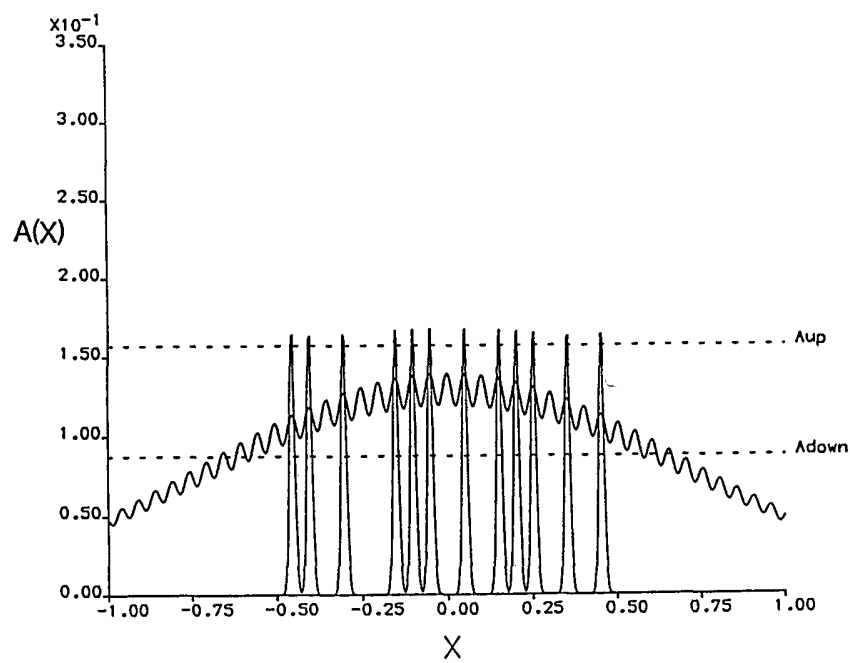

Fig. 5. Our sinusoidally modulated hold beam with respect to the appropriate upswitching and downswitching amplitudes $(M=0.08)$. Encoding of binary patterns on the circulating beam is attained by temporally abrupt superimposition of the addressing Gaussians (here shown in the configuration 1101001110101110101).

hold beam is a sinusoidally modulated Gaussian, Eq. (5). Such a beam may be easily produced experimentally by interference techniques.

$$
A(x)=A_{0}\left(1+M \cos k_{m} x\right) \exp \left(-x^{2}\right) .
$$

The modulation depth is given by $M$, which may assume either sign depending on whether a finite odd or even number of pixel sites is desired. The density of the transverse array scales with the spatial pixelation frequency, $k_{m}$. The most obvious initial constraint on array size is the requirement that pixel locations must be such that the local hold-beam amplitude lies between the appropriate bistable switch points, denoted Aup and Adown in Fig. 5.
These switch levels are higher than those calculated for the smooth beam, since finite $M$ entails additional intensity gradients; the determination of these levels necessitated not only a preliminary switching study of the full set $\left\{F, A_{0}, M, k_{m}\right\}$ but also consideration of address characteristics. $\quad A_{0}$ and peak (Gaussian) address amplitude, $A_{S}$, are parameterized, in a manner similar to that for the twobeam case, as

$$
A_{0}=A_{\text {down }}+C_{H}\left(A_{\text {up }}-A_{\text {down }}\right), \quad A_{S}=C_{S} A_{0} .
$$

To avoid overshoot and critical slowing down, the global maximum of the hold beam is chosen to be approximately $70 \%$ into the working range, requiring that $C_{H} \leq 0.7$. Also shown in Fig. 5 are the address Gaussians, in the configuration 1101001110101110101, which are incorporated into $A(x)$ for a switch duration $T$ (cavity transits). It is now clear that the address beams need to be sufficiently well resolved to avoid induced switching in adjacent sites and, with hindsight, we shall present results here for which $X 0$ is kept fixed at 4.0. The choice of $C_{S}$ depends strongly on that of $T$, because there is a distinct trade-off between switch power and duration. The subtleties of this and other scalings that involve the temporal switching dynamics have been the subject of an extensive study. ${ }^{22}$ In particular, switch-on time has been seen to be a simple and controllable function of address specifications. It has been found that the criterion of performance between the two switch points is not sufficient-marginally stable end pixels can disrupt the stability of the whole array. Unaddressed end pixels seem to permit a buffering of the array from the edge instabilities and allow the hold-beam modulation effectively to tile the central portion of the beam in which the solitons sit.

In the following simulations the hold beam is ramped into position during the first 20 round trips and then held

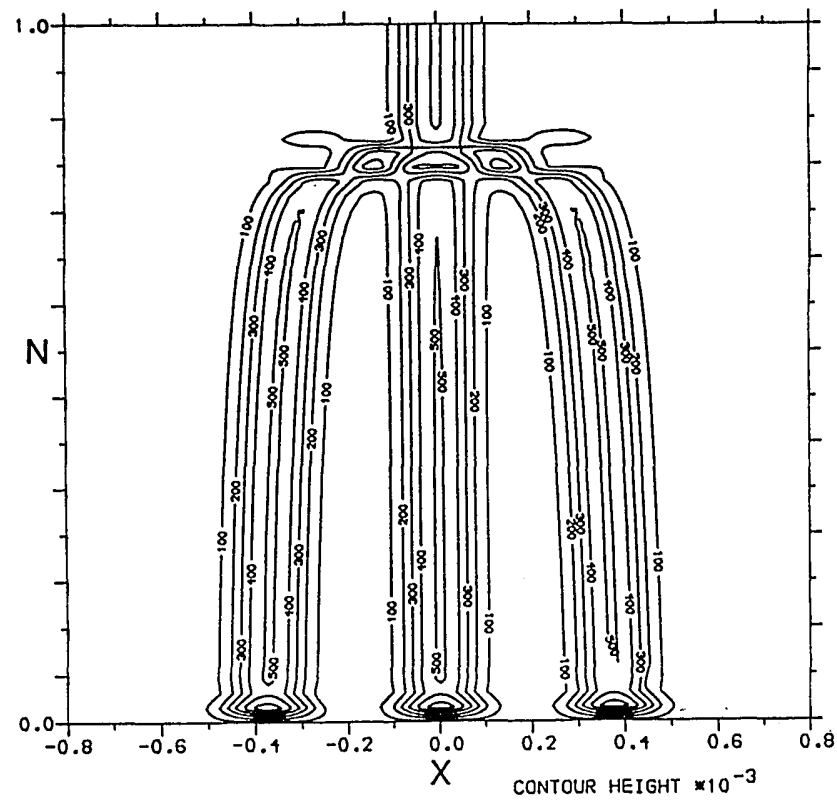

Fig. 6. Simulation shown in Fig. 4 is repeated with an additional (central) induced soliton. Coalescence is at approximately 800 transits. 


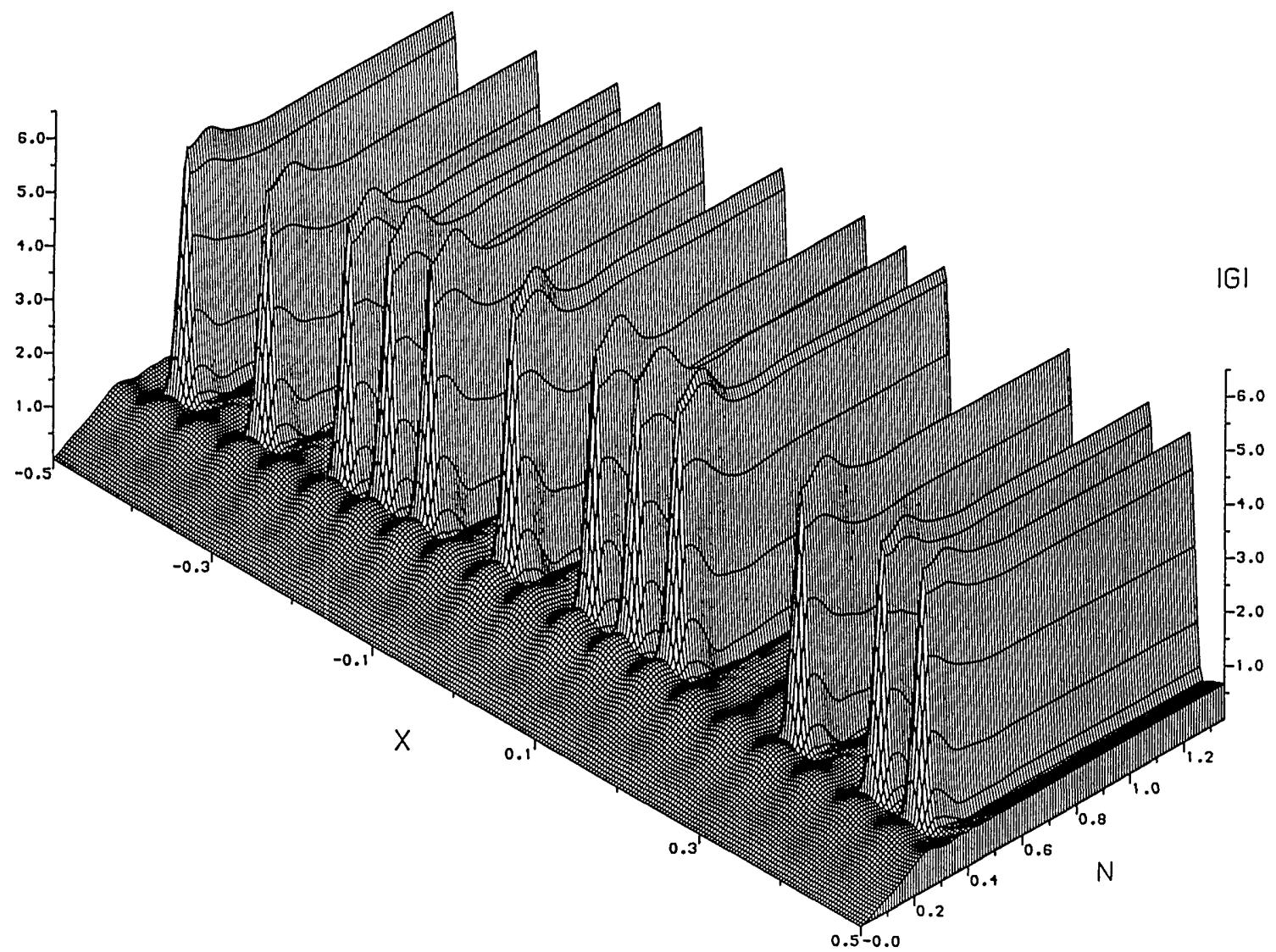

(a)

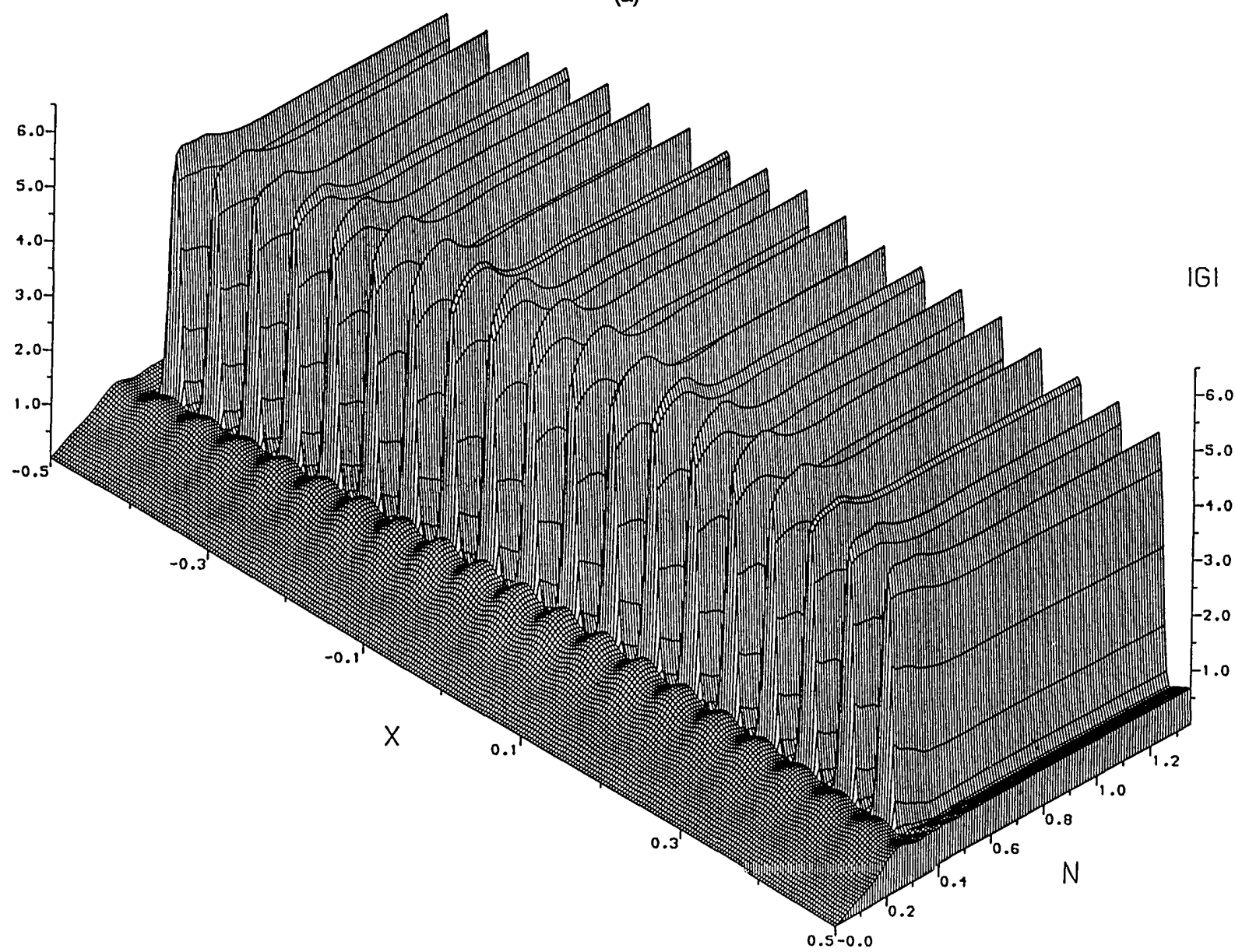

(b)

Fig. 7. Hold-beam initialization and subsequent address of 19-bit patterns. (a) Quasi-random string addressed, $T=5$; (b) Full string of 1 's addressed, $T=1$. $N$ is in units of 100 cavity transits, $|G|$ axis $\times 10^{-1}$. 


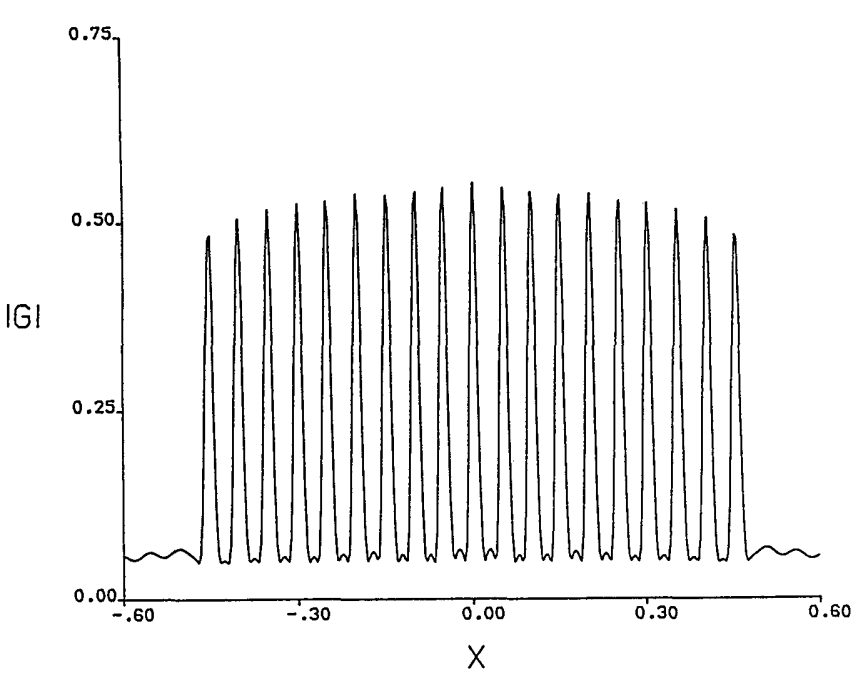

Fig. 8. Overlay of resonator outputs over a period of 2600-3000 cavity transits. In this simulation the hold beam was allowed 30 transits for initialization, and then a full 1111111111111111111 was encoded in the subsequent 5 transits.

$|G|$
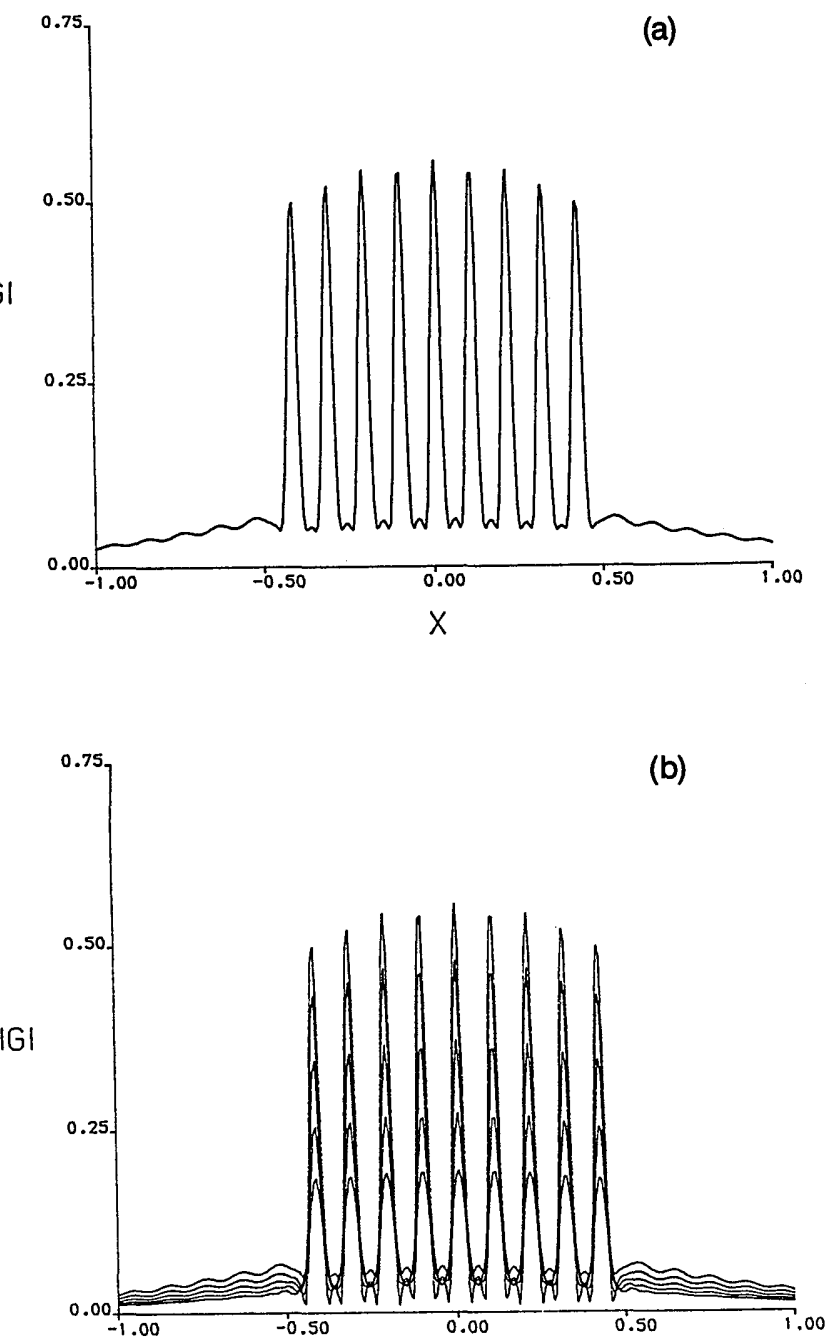

Fig. 9. (a) Overlay of output profiles 800-850 transits after encoding of the string 111111111 on a $F=800$ spot. (b) Beam interrupt, implementing reset during eight subsequent transits. at the operational point for a further 10 trips before the address is implemented.

\section{Spatial Soliton Interaction Forces}

The robust particle nature of solitons and (elastic) multisoliton collisions invokes the concept of interaction forces, which act between each two solitonic centroids and are derived from their own (nonlinear) potentials. Some formalism has been developed for these forces under certain simplifying assumptions, with resultant restricted applicability. Numerical and analytic studies concerning optical soliton interaction have been done mostly in the temporal domain by using or referring to the exact twosoliton solution of the nonlinear Schrödinger equation. ${ }^{23}$ For (resolved) equal-amplitude constituents, the interaction force decreases approximately exponentially with increasing separation while depending sinusoidally on relative phase. ${ }^{24}$ The interaction forces between two solitary pulses may be minimized through a number of techniques, such as using unequal amplitudes, ${ }^{25}$ having relative phases ${ }^{26-28}$ or higher order effects, ${ }^{29}$ and imposing an initial (continuous) phase envelope over the temporal domain. ${ }^{30}$

For our soliton switching, one would expect some qualitative similarities, but here the system is greatly more complicated; it encompasses some of the above effects but also includes host-beam intensity gradients and pixelation (leading to asymmetries and unequal amplitudes), a bistable phase envelope over each pixel, the damping effect of the map, and saturation of the nonlinearity.

To illustrate the existence of interaction forces in the case of diffractive solitons, a periodic plane-wave simulation has been performed. Here a periodic zone in $x$ is considered, within which the backgrounding beam has a plane, rather than Gaussian, spatial distribution. In Fig. 4 an impressive independence was seen between two well-separated solitons. If this integration is repeated but, this time, with an additional (central) soliton, we obtain the results shown in Fig. 6. This corresponds to the first 1000 transits and shows the outer solitons accelerating toward the central one and coalescing after 800 transits. This effect is due solely to the presence of an additional soliton and hence to reduced soliton separation. Coalescence distance may be controlled through specifying the initial separation.

\section{Large Arrays of Soliton Pixels}

Instabilities have imposed upper bounds on spatial pixelation frequencies, which can be used for the array scenarios. When these instabilities occur, their onset time has been found to scale roughly as $\sqrt{F}$. This upper bound on stable $k_{m}$ has been compared with results from an earlier stability analysis, ${ }^{31}$ and its value seems to remain fixed near the low-spatial-frequency edge of the planewave modulational instability band calculated for the maximum bistable output intensity. The most (modulationally) unstable spatial frequencies are far greater than any upper bound imposed on $k_{m}$ through array stability. The simplest step toward interpretation of this observation is in the context of an induced modulational instability-energy balance. ${ }^{21}$

Soliton switching on finite beams has been extended out to a 19-bit array, for which $F=3565$ and $k_{m}=124.4$ 


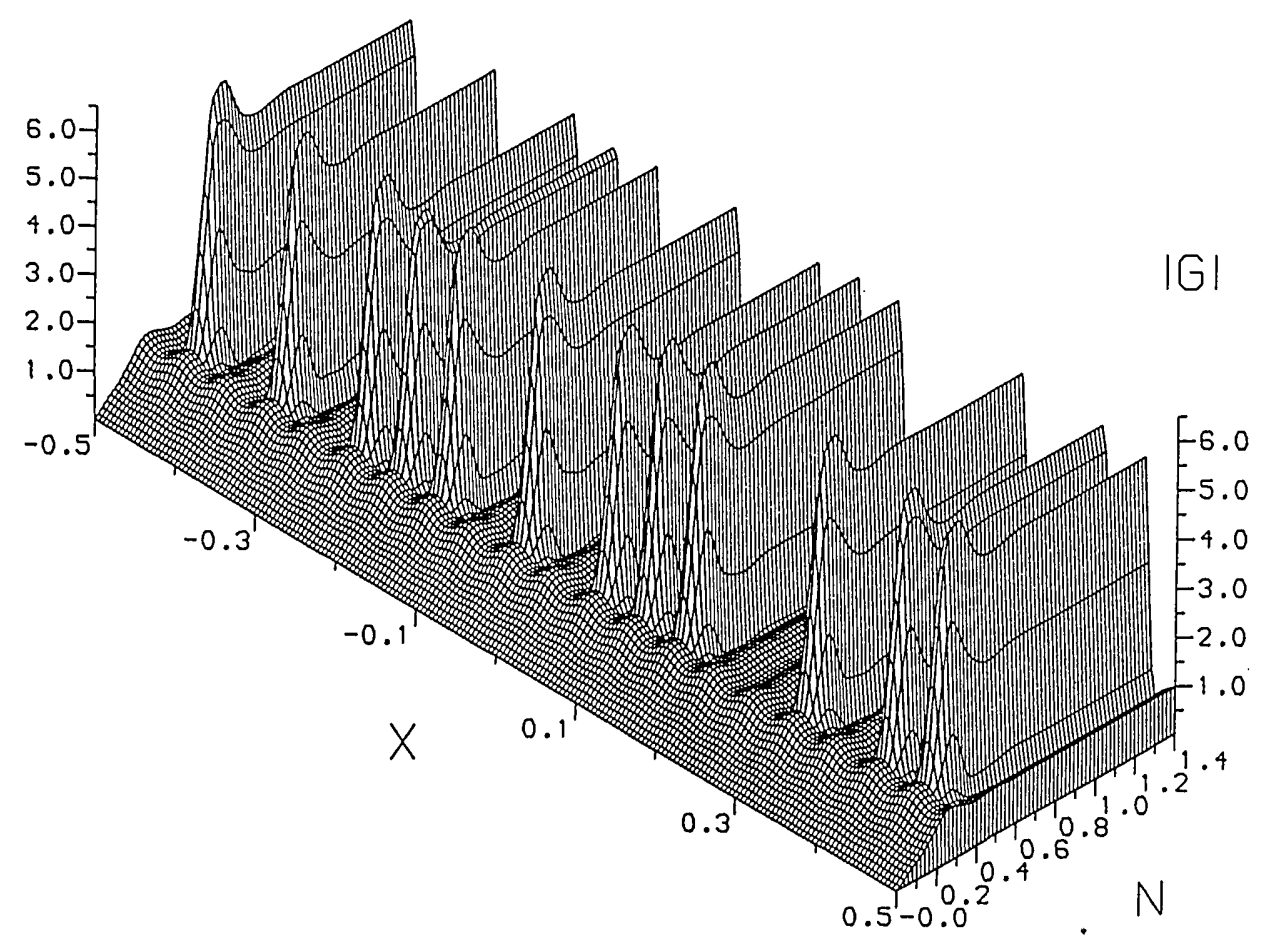

Fig. 10. Encoding of a periodic zone within an infinite-dimensional binary array. Spatial periodicity ensures that this quasi-random pattern recurs indefinitely in both directions of $X$. $N$ is in units of 100 transits, $|G|$ axis $\times 10^{-1}$.

were used. For preservation of numerical accuracy, any further extension would require a prohibitive leap in computational requirements. Figure 7(a) shows hold-beam initialization and subsequent encoding of a quasi-random pattern in five cavity transits, while Fig. 7(b) shows encoding of a full binary string in a single cavity transit. The necessity of using such a large array prompts a few selective trial simulations for multiples of 1000 round trips. The adopted limit was 3000 round trips, and some results are presented in Fig. 8. This shows the final resonator output where the fullest 19-bit address had been encoded and is presented in overlay format for the final 400 cavity transits. This integrity of information storage can also be reproduced when highly asymmetric patterns are chosen.

\section{Wiping the Memory}

Attempts to switch off bistable elements often involve a mechanism similar to beam interrupt. This has been performed for diffusively coupled arrays, ${ }^{32}$ and we have implemented it here. In Fig. 9(a) the string 111111111 is shown stored on a spot of size $F=800$. The process of successfully switching off this pattern, over a period of eight cavity transits, is shown in Fig. 9(b). For this $A_{0}$ was set to zero, and the pattern was allowed to circulate around the cavity, thus experiencing periodic (linear) loss at input and output mirrors. Even in this relatively high-finesse cavity $(R=0.9)$, switch-off by this method requires fewer than ten transit times. The loss is sufficiently rapid to permit a clean switch-off, during which the soliton amplitudes simply drop without any noticeable lateral motion, soliton-soliton interaction, or noisy interference with the adjacent lower branch field. Beam interrupt was performed at the end of most switching simulations, and similar results were seen for all pixel configurations and system parameters used.

This simple, total-wipe strategy is, of course, not ideal. One would like to be able to write a 0 to any pixel site without affecting any other pixels. It is expected that the above results will be equally applicable to local interrupt, but we have found that this operation may be more effectively accomplished through phase-encoded address beams. This phased switching, in fact, gives nearly ideal switching performance, as will be reported elsewhere.

\section{Soliton Addressing in the Limit as $\boldsymbol{F} \rightarrow \infty$}

In the limit $F \rightarrow \infty$, locally, the Gaussian beam becomes planar. To simulate this, and thus to consider the ultimate extension of the foregoing results to essentially an infinite-dimensional pixel array, soliton switching was performed in a periodic plane-wave configuration. In this simulation the narrow address beams, which encode the information, inject a wide and continuous range of frequencies, which permits rapid trapping of diffractive waves into the soliton pixel structures. For finite soliton width, we require finite $F$ in the calculations, so that its role is now only that of specifying the size of a spatial observational window.

In Fig. 10 encoding of a (periodic) zone with a quasirandom string is shown. Necessary address parameters for quick encoding are close to those used on the $F=3565$ spot, indicating that an arbitrarily high number of pixels is possible. Note that spatial periodicity ensures that this quasi-random pattern recurs indefinitely in both directions of $X$, and thus we are simulating a periodic bit pattern in an infinite-dimensional binary array. 


\section{SUMMARY}

Transverse effects permit and constrain information storage in bistable optical systems. Here we have examined, for the first time to our knowledge, diffractively coupled arrays of bistable elements or pixels. Spatial (bright) solitons have an innate appeal as natural pixels, and we have shown that it is possible to devise a soliton memory in which a soliton may be present or absent at each pixel site, giving maximum information capacity. We have reported some results on two-beam interaction and soliton-soliton interactions in a ring cavity containing a self-focusing medium that underpins the array simulations.

It is not sufficient that information can be retained in a memory; it must also be possible to write to and read from this memory. The latter is trivial in our case, because the transmitted beam gives a continuous readout. We have shown that soliton pixels can be turned on rapidly by appropriate address pulses and cleanly wiped by beam interrupt.

\section{ACKNOWLEDGMENTS}

This work was supported in part under a Twinning contract of the European Community. G. S. McDonald is grateful for support from the University of Strathclyde and the U. K. Science and Engineering Research Council.

\section{REFERENCES}

1. S. M. Jensen, IEEE J. Quantum Electron. QE-18, 1580 (1982).

2. S. R. Friberg, A. M. Weiner, Y. Silberberg, B. G. Sfez, and P. S. Smith, Opt. Lett. 13, 904 (1988).

3. P. Li Kam Wa, J. E. Sitch, N. J. Mason, J. S. Roberts, and P. N. Robson, Electron. Lett. 21, 26 (1985).

4. N. J. Doran and D. Wood, J. Opt. Soc. Am. B 4, 1843 (1987).

5. N. J. Doran and D. Wood, Opt. Lett. 13, 56 (1988).

6. K. J. Blow, N. J. Doran, and B. K. Nayar, Opt. Lett. 14, 754 (1989).
7. S. Trillo, S. Wabnitz, E. M. Wright, and G. I. Stegeman, Opt. Lett. 13, 672 (1988).

8. S. Trillo, S. Wabnitz, E. M. Wright, and G. I. Stegeman, Opt. Commun. 70, 166 (1989).

9. H. Adachihara, D. W. McLaughlin, J. V. Moloney, and A. C. Newell, J. Math. Phys. 29, 63 (1988).

10. A. Barthelemy, S. Maneuf, and C. Froehly, Opt. Commun. 55, 201 (1985).

11. S. Maneuf, R. Descilly, and C. Froehly, Opt. Commun. 65, 193 (1988).

12. J. S. Aitchison, A. M. Weiner, Y. Silberberg, M. K. Oliver, J. L. Jackel, D. E. Leaird, E. M. Vogel, and P.W. E. Smith, Opt. Lett. 15, 471 (1990).

13. E. Abraham, C. Godsalve, and B. S. Wherrett, J. Phys. 49, 43 (1988).

14. A. K. Kar, R. M. Harris, G. S. Buller, S. D. Smith, and A. C. Walker, J. Phys. 49, 443 (1988).

15. W. J. Firth and I. Galbraith, IEEE J. Quantum Electron. QE-21, 1399 (1985).

16. D. J. Hagan, H. A. Mackenzie, H. A. Al-Attar, and W. J. Firth, Opt. Lett. 10, 187 (1985).

17. K. Tai, J. V. Moloney, and H. M. Gibbs, Opt. Lett. 7, 429 (1982).

18. K. Ikeda, Opt. Commun. 30, 257 (1979).

19. R. H. Hardin and F. D. Tappert, SIAM Rev. 15, 423 (1973).

20. J. V. Moloney and H. M. Gibbs, Phys. Rev. Lett. 48, 1607 (1982).

21. G. S. McDonald and W. J. Firth, "All-optical switching in a nonlinear resonator," J. Mod. Opt. (to be published).

22. G. S. McDonald, "Spatial solitary wave optical memory," Ph.D. dissertation (Strathclyde University, Glasgow, 1989).

23. V. E. Zakharov and A. B. Shabat, Sov. Phys. JETP 34, 62 (1972).

24. J. P. Gordon, Opt. Lett. 8, 596 (1983).

25. P. L. Chu and C. Desem, Electron. Lett. 21, 1133 (1985).

26. B. Hermansson and D. Yerick, Electron. Lett. 19, 570 (1983).

27. E. Shiojiri and Y. Fujii, Appl. Opt. 24, 358 (1985).

28. D. Anderson and M. Lisak, Opt. Lett. 11, 174 (1986).

29. P. L. Chu and C. Desem, Electron. Lett. 21, 228 (1985).

30. E. M. Dianov, Z. S. Mikonova, and V. N. Serkin, Sov. J. Quantum Electron. 16, 1148 (1986).

31. D. W. McLaughlin, J. V. Moloney, and A. C. Newell, Phys. Rev. Lett. 7, 681 (1985).

32. I. Galbraith, "Diffractive and diffusive phenomena in optically bistable devices," Ph.D. dissertation (Heriot-Watt University, Edinburgh, 1985). 